\title{
High-gravity central stars
}

\author{
Thomas Rauch \\ Institut für Astronomie und Astrophysik, Universität Tübingen, Germany \\ email: rauch@astro.uni-tuebingen.de
}

\begin{abstract}
NLTE spectral analyses of high-gravity central stars by means of state-of-the-art model atmosphere techniques provide information about the precursor AGB stars. The hydrogendeficient post-AGB stars allow investigations on the intershell matter which is apparently exhibited at the stellar surface. We summarize recent results from imaging, spectroscopy and spectropolarimetry.
\end{abstract}

Keywords. stars: abundances, stars: AGB and post-AGB, stars: atmospheres, stars: early-type, stars: evolution, stars: individual (KPD 0005+5106, LS V+4621, PG 0109+111, PG 1034+001), white dwarfs, planetary nebulae: individual (Abell 36, Abell 78, EGB 5, He 2-36, K 1-16, LSS 1362, NGC 1360, NGC 2371, Sh 2-216)

\section{Introduction}

High-gravity central stars (CS) of planetary nebulae (PNe) display the hottest stage of stellar post-AGB evolution just at the entrance of the white-dwarf cooling track on the way towards the stellar graveyard. These stars are in a short phase between decreasing mass loss and thus, less spectral contamination by features which are formed under the influence of strong stellar winds (cf. Kudritzki, these proceedings), and increasing gravity which, in interplay with radiative levitation, will wipe out all photospheric information about their progenitors.

We will show progress in analyses of high-gravity CSPNe and demonstrate that spectral analyses by means of non-LTE model-atmosphere techniques (Sect. 2) provide a powerful tool to investigate the properties of these stars and to determine constraints for evolutionary theory (Sect. 2.3).

\section{Imaging and spectroscopy}

High-gravity post-AGB stars, i.e. hot, compact stars, have arrived at extremely high effective temperatures $\left(T_{\text {eff }}\right.$ up to $\left.\approx 200 \mathrm{kK}\right)$. Thus it appears that their flux maximum is located in the EUV spectral range and they are intrinsically faint in the optical. For a reliable spectral analysis, the evaluation of ionization equilibria is necessary for the determination of $T_{\text {eff }}$ (see Sect.6). Since the strongest metal lines are found almost exclusively in the high-energy range, its accuracy is dependent on the availability of high-resolution and high-S/N spectra in the UV wavelength range. These have been provided e.g. by the Hubble Space Telescope (HST) with the Faint Object Spectrograph (FOS) and the Space Telescope Imaging Spectrograph (STIS), and by the Far Ultraviolet Spectroscopic Explorer (FUSE). Examples for analyses which have been performed based on data obtained with these are given in Sections 2.1 and 2.3. 
Table 1. Satellite instruments used for spectral analyses of high-gravity CS.

\begin{tabular}{lllrr}
\hline satellite & instrument & period & \multicolumn{1}{c}{$\lambda / \AA$} & resolution \\
\hline HST & FOS & $1990-1997$ & $1150-8000$ & $<1300$ \\
HST & STIS & $1997-2004$ & $1150-3175$ & $<114000$ \\
FUSE & & $1999-$ & $905-1185$ & $\approx 20000$ \\
\hline
\end{tabular}

\section{1. $L M C+S M C$ central stars}

In order to constrain the initial-to-final mass relation, analyses of 35 LMC (Villaver et al. 2003) and 27 SMC (Villaver et al. 2004) CS have been performed, based on imaging and spectroscopy by HST STIS and the Wide-Field Planetary Camera (WFPC2). A comparison in the Hertzsprung-Russell diagram with evolutionary tracks of Vassiliadis \& Wood (1994) for LMC and SMC metallicities yields average CS masses of $0.65 \mathrm{M}_{\odot}$ and $0.63 \mathrm{M}_{\odot}$, respectively. Although these analyses are free of distance uncertainty, the determination of $T_{\text {eff }}$ by the Zanstra method imposes an imponderable error which one has to be aware of.

An analysis of seven LMC CS has been presented by Herald \& Bianchi (2004). They used FUSE and HST (FOS + STIS) spectra and employed NLTE model-atmosphere techniques using the codes TLUSTY (Hubeny \& Lanz 1995) and, since most objects display wind features $\left(\dot{M} \approx 5 \times 10^{-8} \mathrm{M}_{\odot} \mathrm{yr}^{-1}\right)$, CMFGEN (Hillier \& Miller 1998, 1999). The CS's $T_{\text {eff }}$ range from 38 to $70 \mathrm{kK}$ and five of them have LMC abundances. Since the surface gravity $g$ could not be determined from the UV spectra and adequate optical spectra are not available, it is adopted within $4.3 \leqslant \log g \leqslant 5.3(\mathrm{cgs})$ in order to investigate errors in the parameter determination. However, this analysis does not represent the standard of modern spectral analysis because $g$ is not self-consistently determined. One has to be aware of this uncertainty, although this analysis aims at a characterization of PNe through a better understanding of the CS $\leftrightarrow$ PN system and of wind properties.

\subsection{Hydrogen-rich central stars}

Newly available high-resolution UV spectra, obtained by HST STIS and FUSE, enabled Traulsen et al. (2005) to establish an improved temperature scale for seven hot hydrogenrich CSPN. Within their NLTE model-atmosphere analysis, they evaluated the ionization balances of $\mathrm{C}, \mathrm{N}$, and $\mathrm{O}$ which show rich line spectra in the $\mathrm{UV}$, in order to derive $T_{\text {eff }}$ precisely. Hoffmann et al. (2005) extended this analysis to the use of the Fe VI/Fe VII ionization equilibrium.

In an on-going analysis of LS V+4621 (Ziegler et al. 2006), all iron-group elements are considered individually. In Sect. 6, we show some details of this state-of-the-art spectral analysis.

\subsection{Hydrogen-deficient central stars}

While hydrogen-rich stellar post-AGB evolution is quite well understood, the picture for hydrogen-deficient evolution (about $20 \%$ of all post-AGB stars) became clearer only in the last few years (cf. Herwig, these proceedings). Spectral analysis of these objects has placed challenges again and again to evolutionary theory and, vice versa, predictions from evolutionary models have been verified by spectral analysis.

One example is the discovery of the NeVII $\lambda 3643.6 \AA$ absorption line in the optical spectra of three extremely hot PG 1159-type CSPN (Werner \& Rauch 1994). An abundance analysis with NLTE model atmospheres has shown that this line can be reproduced with a Ne abundance of $\approx 2 \%$ (by mass) in the photosphere. Early calculations of Iben 


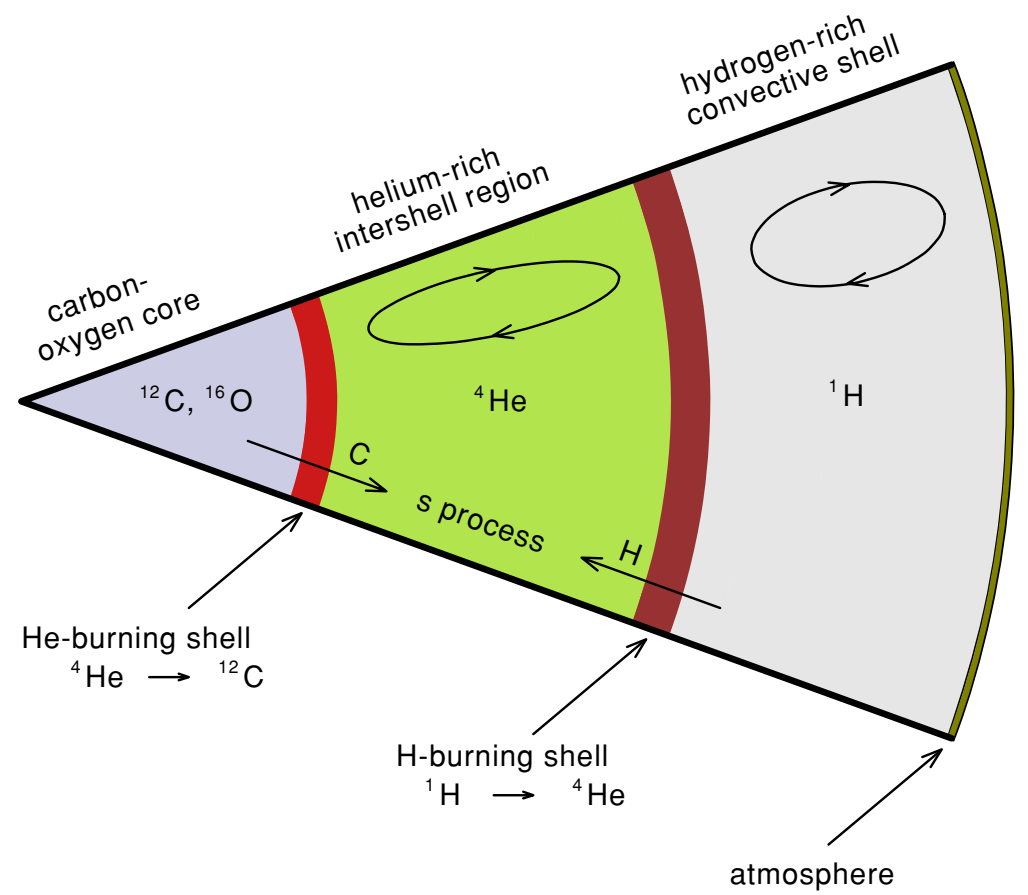

Figure 1. Interior of an AGB star. Between the He- and H-burning shells, the so-called intershell matter is located $\left(\approx 10^{-2} \mathrm{M}_{\odot}\right)$. The H-rich envelope $\left(\approx 10^{-4} \mathrm{M}_{\odot}\right)$ prohibits an insight into the intershell matter. A (V)LTP may lay bare this matter.

\& Tutukov (1985) could not explain the observation, although they predicted Ne to be the fourth most abundant element $(\approx 2 \%$ mass fraction, following $\mathrm{C}, \mathrm{O}$, and $\mathrm{He}$ ) just below the helium-burning shell - inaccessible for spectroscopy. New evolutionary models by Herwig et al. (1999), which consider a realistic overshoot adopted from hydrodynamical simulations, predict a mass fraction of about $3.5 \%$ of $\mathrm{Ne}$ in the intershell (Fig. 1), in good agreement with the spectroscopic result. The recent identification of the Ne VII $\lambda 973.3 \AA$ absorption line in FUV (Werner at al. 2004b) spectra (obtained with FUSE) of six PG 1159 stars, the presence of Ne VII $\lambda 3643.6 \AA$ in eight and of Ne VII $\lambda \lambda 3850-3910 \AA$ in four PG 1159 stars (Werner at al. 2004b) corroborates these evolutionary models. Stellar atmospheres with a content of $2 \%$ of Ne (eleven times solar) can reproduce well the observed Ne VII lines. It its worthwhile to note that Herald et al. (2005) have shown that the Ne VII $\lambda 973 \AA$ line appears with a strong P Cygni wind profile in the CS of the PNe NGC 2371, Abell 78, and K 1-16 (this is much stronger than the supposed C III $\lambda 977 \AA$ $\mathrm{P}$ Cygni feature in these CS).

Another example is the reproduction of the CHANDRA spectrum of the hydrogen- and helium-deficient star H 1504+65 (Werner at al. 2004a). The consideration of magnesium with a mass fraction of $2 \%$ significantly improved the spectral fit. For $\mathrm{Mg}$, Iben \& Tutukov (1985) predicted an equal amount to that of Ne, also just below the helium-burning shell.

The reason for our special interest in hydrogen-deficient post-AGB stars, especially in the PG 1159 stars, lies in the fact that these stars experience a (very) late thermal pulse (cf. Herwig, these proceedings) during their evolution which mixes the entire hydrogen-rich envelope (Fig. 1) into the intershell matter where hydrogen is then burned. Spectroscopy of these stars provides a direct view of the former intershell matter and allows one to conclude on details of nuclear and mixing processes in AGB stars. 
We will now briefly summarize PG 1159 abundance determinations based on recent HST and FUSE spectroscopy. He, C, N, O, Ne, Mg, F (Sect. 2.3.2), and Si are in line with predictions from evolutionary models (Reiff et al. 2005 and these proceedings, and Jahn et al. 2006). Fe (Sect. 2.3.1) shows a surprisingly large depletion, as well as S (Sect. 2.3.3). $\mathrm{P}$ is predicted to be overabundant by a factor of up to 25 (still uncertain) but is found to be roughly solar (Jahn et al. 2006).

For a more detailed review on elemental abundances in bare CSPN, see Werner \& Herwig (2006) and references therein.

\subsubsection{Iron}

Evolutionary calculations predict a reduced, i.e. sub-solar, Fe intershell abundance due to n-captures in the s-process. The FUSE wavelength range covers the strongest Fe VII lines (this is the dominant ionization stage in hot PG 1159 stars). Up to now, FUSE spectra of three PG 1159 stars with sufficiently high S/N have been analyzed but no iron lines are detectable. Thus, a very strong Fe depletion (at least 1-2 dex) takes place in the intershell (Miksa et al. 2002).

\subsubsection{Fluorine}

The first discovery of fluorine (F VI $\lambda 1139.50 \AA$ ) in FUSE spectra of hot post-AGB stars (Werner at al. 2005) has shown that AGB stars are efficiently synthesizing fluorine which even survives a (V)LTP - abundances of up to $200 \times$ solar have been determined in PG 1159 stars. Measurements in PNe (Zhang \& Liu, these proceedings) have found that $\mathrm{F}$ is generally overabundant in $\mathrm{PNe}$, thus providing new evidence for its synthesis in AGB stars.

\subsubsection{Sulfur}

The photospheric abundance of sulfur should not be changed by nuclear processes. A recent spectral analysis of PG 1159-035 (Jahn et al. 2006) has shown, however, that S is strongly underabundant (Fig. 2).

\section{Spectropolarimetry}

Recently, Jordan et al. (2005) reported on the first directly measured (via spectropolarimetry with the Focal Reducer and low dispersion Spectrograph (FORS1) attached to the Very Large Telescope (VLT)) magnetic fields ( $B$ of the order of $\mathrm{kG}$ ) in four CS (of the PNe NGC 1360, EGB 5, LSS 1362, A 36). From these field strengths (under the assumption of complete flux conservation), they estimate that the main-sequence precursors had field strengths of the order of some G. The later white dwarfs (WDs) will have fields of some MG. Many WDs with such strong fields are indeed known. However, the influence of magnetic fields on the morphology of PNe is still under debate and this first measurement is a valuable help to quantify their impact in magneto-hydrodynamical modeling.

\section{Imaging}

PNe are detected only around every other PG 1159 star. Depending on their individual evolution, the PNe which have been ejected on the AGB have most likely dispersed below the detection limit. Over the last years we spent some time on a search for nebular emission around PG 1159 stars and related objects. Our search has been entirely negative, except for the DO white dwarf PG0109+111 (Werner et al. 1997) where a possible PN 


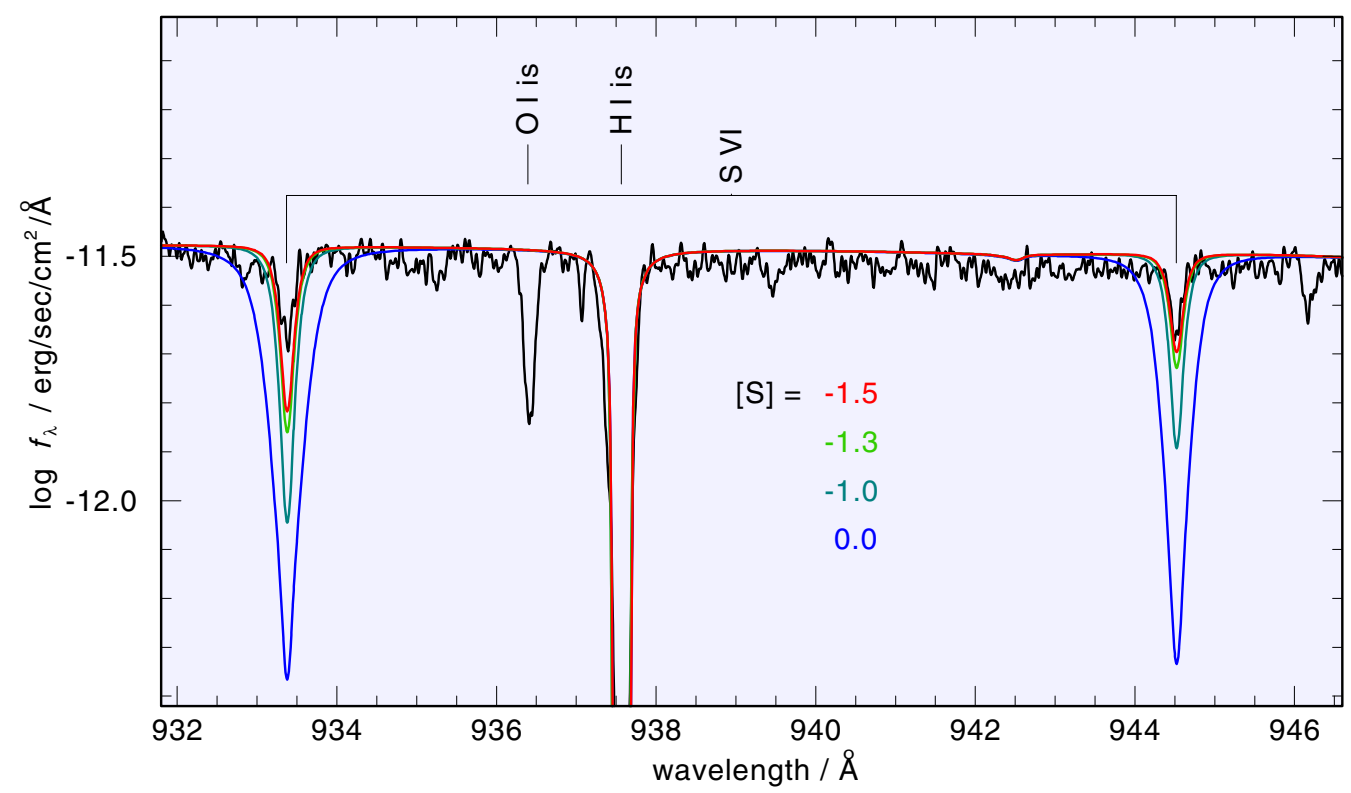

Figure 2. Section of the STIS spectrum of PG 1159-035 showing the S vi resonance doublet. Obviously, $\mathrm{S}$ is strongly underabundant (about 0.02 of the solar abundance).

was found with a diameter of $0.8 \mathrm{pc}$. Recently, Hewett et al. (2003) discovered a PN (Hewett 1) around the DO PG 1034+001 with a diameter of 3.5-7.0 pc. An investigation of data from the Southern H-Alpha Sky Survey Atlas (SHASSA, Gaustad et al. 2001) by Rauch et al. (2004) has shown that an enormous ionized halo exists around this object with a diameter of 16-24 pc. An ionized nebula has also been found by Chu et al. (2004) around the DO KPD 0005+5106. Since the ionized mass is about $70 \mathrm{M}_{\odot}$ this is unambiguously ambient interstellar medium (ISM). Chu et al. (2004) also suggested that the nebula around PG 1034+001 is simply ionized ISM. Frey (these proceedings) also expects this for PG 0109+111's nebula. Although all these nebulosities are probably not PNe, the importance of hot white dwarfs as ionizing sources for the ISM is quite evident.

\section{Evolutionary Theory}

Recently, new calculations for post-AGB evolution have been presented by Althaus et al. (2005a) (hydrogen-deficient non-DA white dwarfs), Althaus et al. (2005b) (DA white dwarfs), Miller Bertolami et al. (2006) (born-again scenario), and Miller Bertolami \& Althaus (2006) (PG 1159 and $\mathrm{O}(\mathrm{He})$ stars). These new calculations should be used for, e.g., the determination of white-dwarf masses (Herwig, priv. comm.). Miller Bertolami \& Althaus (2006) find a systematic offset of $\Delta M \approx-0.05 \mathrm{M}_{\odot}$ for the known PG 1159 stars compared to the values summarized by Werner \& Herwig (2006) which had been determined from older evolutionary calculations.

\section{FUSE and HST spectroscopy of LS V+4621}

$\mathrm{LSV}+4621$ is the exciting star of Sh $2-216$. This is the closest $(d=130 \mathrm{pc}) \mathrm{PN}$ known, with an apparent diameter of 1.6 degrees projected at the sky. High resolution FUV and UV spectra (FUSE: $67.6 \mathrm{ksec}$ in 2003/2004, $\Delta \lambda \approx 0.05 \AA$, STIS: 5.5 ksec in $2000, \Delta \lambda \approx 0.06 \AA$ ) are available. These have been analyzed by means of NLTE 


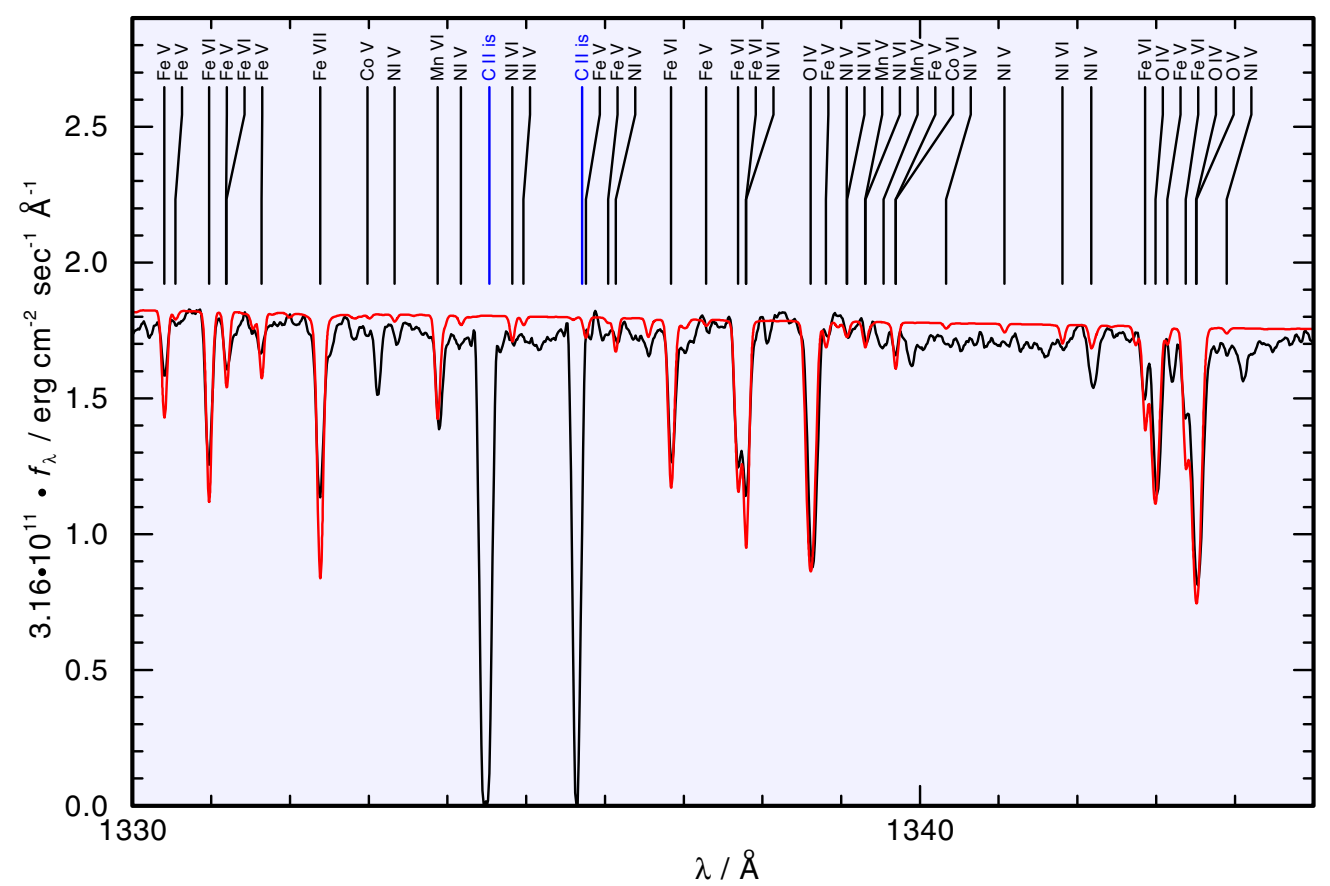

Figure 3. Section of the HST STIS spectrum of LSV+4621 (shifted by $v_{\mathrm{rad}}=20.386 \mathrm{~km} / \mathrm{sec}$ in order to match the rest wavelengths of the photospheric lines) compared with the theoretical spectrum ( $T_{\text {eff }}=95 \mathrm{kK}, \log g=6.9$, abundances see Fig. 4). The identified lines are marked. 'is' denotes lines of interstellar origin. Note that e.g. lines from Fe V, Fe VI, and Fe VII are matched simultaneously. For the calculation of the synthetic spectrum, Kurucz's POS line lists (Kurucz 1996), which contain lines with laboratory measured wavelengths only, are used. For the model-atmosphere calculation Kurucz's LIN line lists are used (including all theoretically calculated lines in addition). This may explain the existing differences between observation and model, e.g. unidentified features.

model-atmosphere techniques. We employed TMAP, the Tübingen NLTE Model Atmosphere Package (Werner et al. 2003) in order to calculate models which consider $\mathrm{H}+\mathrm{He}+\mathrm{C}+\mathrm{N}+\mathrm{O}+\mathrm{Mg}+\mathrm{Si}$ by "classical" model atoms and $\mathrm{Ca}-\mathrm{Ni}$ in a statistical approach (Rauch \& Deetjen 2003) introducing superlevels and superlines. In total, 531 levels are treated in NLTE, with 1761 individual lines and about nine million iron-group lines (Kurucz 1996).

The HST STIS spectrum allows one to determine the interstellar neutral hydrogen column density $N_{\mathrm{HI}}=1.10 \pm 0.05 \cdot 10^{20} \mathrm{~cm}^{-2}$ and the interstellar extinction $E_{\mathrm{B}-\mathrm{V}}=$ 0.03. The evaluation of the ionization equilibria of $\mathrm{N}$ IV / N v, O IV / O v, Si IV / Siv, $\mathrm{FeV} / \mathrm{FeVI} / \mathrm{FeVII}$, and Niv/ Nivi allows the determination of $T_{\text {eff }}=95 \pm 3 \mathrm{kK}$ and $\log g=6.9 \pm 0.2(\mathrm{cgs})$ with unprecedented precision.

Siv has been newly identified. Mg IV lines have been discovered for the first time in these objects (Ziegler et al. 2006). A comparison of the synthetic model with the observations is shown in Fig.3. The determined abundances are summarized in Fig. 4.

\section{NLTE model-atmosphere fluxes}

Recent progress in observational techniques as well as numerical methods facilitates the close examination of both PNe and their central stars. NLTE model-atmosphere calculations have arrived at a high level of sophistication, providing reliable ionizing 


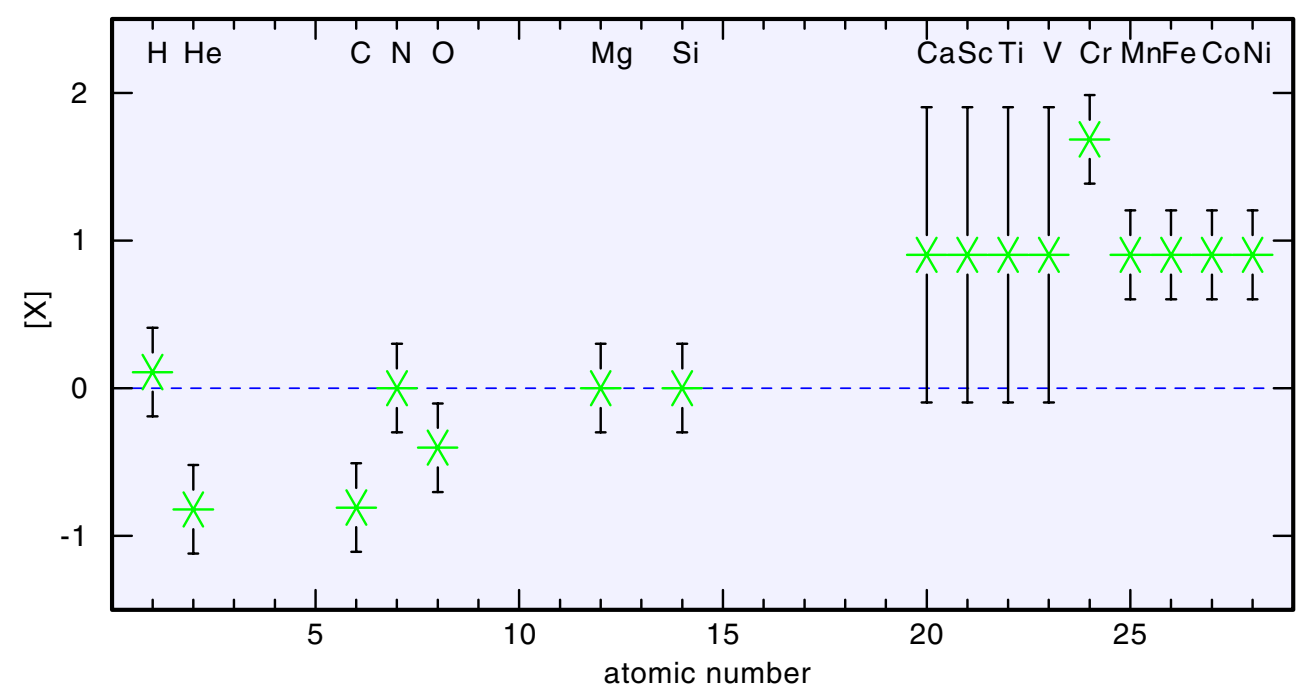

Figure 4. Photospheric abundances in LS V+4621. [X] denotes log (abundance / solar abundance). The abundance pattern indicates that gravitational settling is already efficient for $\mathrm{He}$ while radiative levitation causes strong overabundances of the iron-group elements. The large error ranges for $\mathrm{Ca}, \mathrm{Sc}, \mathrm{Ti}$, and $\mathrm{V}$ are due to the fact that no Kurucz POS lines can be identified in the STIS spectrum. Since the FUSE spectrum is strongly contaminated by interstellar absorption, the abundance determination for these elements is still less certain.

fluxes to be used to construct adequate photoionization models of PNe. The use of a blackbody approximation instead appears attractive because it is so easy to use. However, a challenge for the next few years is to improve PNe modeling with a reliable consideration of the central stars' fluxes.

Grids of flux tables calculated for high-gravity CS and a variety of photospheric chemical compositions can be retrieved from http://astro. uni-tuebingen.de/ rauch. These can be used as ionizing fluxes in photoionization codes such as CLOUDY (Ferland 2003) or MOCASSIN (cf. Ercolano these proceedings). In case of any special requirements please do not hesitate to ask the author for an individual model.

\section{Acknowledgements}

I would like to thank Klaus Werner for careful reading of the manuscript. This research was supported by the DLR under grant 50 OR 0201 and by a grant of the DFG (KON 218/2006). This research has made use of NASA's Astrophysics Data System and of the SIMBAD Astronomical Database, operated at CDS, Strasbourg, France.

\section{References}

Althaus, L.G., Serenelli, A.M., Panei, J.A., Córsico, A.H., García-Berro, E., \& Scóccola, C.G. 2005a, A\&A 435, 631

Althaus, L.G., Miller Bertolami, M.M., Córsico, A.H., García-Berro, E., \& Gil-Pons, P. 2005b, $A \mathscr{E} A 440, \mathrm{~L} 1$

Chu, Y.-H., Gruendl, R.A., Williams, R.M., Gull, T.R., \& Werner, K. 2004, AJ 128, 2357

Ferland, G. 2003, ARA $\& A$ 41, 517

Gaustad, J.E., McCullough, P.R., Rosing, W., \& Van Buren, D. 2001, PASP 113, 1326

Herald, J.E., \& Bianchi, L. 2004, ApJ 611, 294

Herald, J.E., Bianchi, L., \& Hillier, D.J. 2005, ApJ 627, 424

Herwig, F., Blöcker, T., Langer, N., \& Driebe, T. 1999, A $\& A$ 349, L5 
Hewett, P.C., Irwin, M.J., Skillman, E.D., Foltz, C.B., Willis, J.P., Warren, S.J., \& Walton, N.A. 2003, ApJ 599, L37

Hillier, D.J., \& Miller D.L. 1998, ApJ 496, 407

Hillier, D.J., \& Miller D.L. 1999, ApJ 519, 354

Hoffmann A.I.D., Traulsen I., Dreizler S., Rauch T., Werner K., \& Kruk J.W. 2005, The ASP Conference Series Vol. 344 (San Francisco), p. 312

Hubeny, I., \& Lanz, T. 1995, ApJ 439, 875

Iben, I.Jr., \& Tutukov, A.V. 1985, ApJS 58, 661

Jahn, D., Rauch, T., Reiff, E., Werner, K., Kruk, J.W., \& Herwig, F. 2006, A $\& A$, submitted

Jordan, S., Werner, K., O'Toole, S.J. (2005), A\& A 432, 273

Kurucz, R.L. 1996, IAU Symp. 176, Kluwer, Dordrecht, 52

Miksa, S., Deetjen, J.L., Dreizler, S., Kruk, J.W., Rauch, T., \& Werner, K. 2002, A $\& A$ 389, 953

Miller Bertolami, M.M., Althaus, L.G., Serenelli, A.M., \& Panei, J.A. 2006, A\&A 449, 313

Miller Bertolami, M.M., \& Althaus, L.G. 2006, A\&A, 454, 845

Rauch, T., \& Deetjen, J.L. 2003, ASP Conference Series Vol. 288 (San Francisco), p. 103

Rauch T., Kerber F., \& Pauli E.-M. 2004, A\& A 417, 647

Reiff S., Rauch T., Werner K., \& Kruk J.W. 2005, ASP Conference Series Vol. 344 (San Francisco), p. 225

Traulsen I., Hoffmann A.I.D., Rauch T., Werner K., Dreizler S., \& Kruk J.W. 2005, ASP Conference Series Vol. 344 (San Francisco), p. 325

Vassiliadis, E., \& Wood, P.R. 1994, ApJS 92, 125

Villaver, E., Stanghellini, L., \& Shaw, R.A. 2003, ApJ 597, 298

Villaver, E., Stanghellini, L., \& Shaw, R.A. 2004, ApJ 614, 716

Werner, K., \& Rauch, T. 1994, A\& A 284, L5

Werner, K., \& Herwig, F. 2006, PASP 118, 183

Werner K., Bagschik K., Rauch T., \& Napiwotzki R 1997, A\&A 327, 721

Werner, K., Dreizler, S., Deetjen, J.L., Nagel, T., Rauch, T., \& Schuh, S.L 2003, ASP Conference Series Vol. 288 (San Francisco), p. 31

Werner, K., Rauch, T., Barstow, M.A., \& Kruk, J.W. 2004a, A\&A 421, 1169

Werner, K., Rauch, T., Reiff, E., Kruk, J.W., \& Napiwotzki, R. 2004b, A $\& A$ 427, 685

Werner, K., Rauch, T., \& Kruk, J.W. 2005, A\&\&A 433, 641

Ziegler, M., Rauch, T., Werner, K. $2006 A \& A$, in prep. 